\title{
Study of variation of gravitational constant (G) in very strong gravitational field
}

\author{
Dipo Mahto ${ }^{1}$, Md Shams Nadeem ${ }^{2}$, Umakant Prasad ${ }^{3}$, Kumari Vineeta ${ }^{4}$ \\ ${ }^{1}$ Department of Physics, Marwari College, T.M.B.U. Bhagalpur-812007, India \\ ${ }^{2}$ Department of Physics, T.M.B.U. Bhagalpur-812007, India \\ ${ }^{3}$ Department of Physics, T. N. B. College, T.M.B.U. Bhagalpur-812007, India \\ ${ }^{4}$ Department of Education, S.M. College, Bhagalpur, India
}

\section{Email address:}

dipomahto@hotmail.com(D. Mahto),msn.phy@gmail.com (M.S. Nadeem),umakant.prasad@gmail.com(U. Prasad) vineeta.priyadarshi@gmail.com (K. Vineeta)

\section{To cite this article:}

Dipo Mahto, Md Shams Nadeem, Umakant Prasad, Kumari Vineeta. Study of Variation of Gravitational Constant (G) in the Very Strong Gravitational Field. International Journal of Astrophysics and Space Science. Vol. 1, No. 4, 2013, pp. 56-60.

doi: $10.11648 /$ j.ijass.20130104.17

\begin{abstract}
In the present work, we have derived the formula for the variation of the gravitational constant given by $G^{\prime}=G / \sqrt{1-v^{2} / c^{2}}$ in very strong gravitational field of the compact bodies like super massive black holes and neutron stars applying special relativity and Newton's law of gravitation for two bodies where $\mathrm{v}$ be the velocity of spinning compact bodies like black holes, neutron stars etc. and $\mathrm{c}$ be the velocity of light and calculated the variability of the gravitational constant $(\mathrm{G})$ at different speed of the spinning of the black holes, neutron stars and quasars to show the speed is also factor governing the force of gravity addition to the mass.
\end{abstract}

Keywords: Compact Body, Gravitational Constant, Black Hole and Neutron Star

\section{Introduction}

Isaac Newton proposed Universal law of Gravitation in 1687 , which states that every particle in the universe exerts a force on every particle along the line joining their centres. The magnitude of the force is directly proportional to the product of the masses of the two particles and inversely proportional to the square of the distance between them (Newton, 1687).

In 1798, Cavendish measured $G$ implicitly; using a torsion balance invented by the geologist Rev. John Michell and calculated implies a value for $G$ of $6.754 \times 10^{-11} \mathrm{~m}^{3} \mathrm{~kg}^{-1}$ $\mathrm{s}^{-2}$ (Brush et al. 2001).

In 1915, Albert Einstein demonstrated better theory of gravitation on the basis of general relativity, which has overcome the limitations of Newton's law of universal gravitation (Bergmann, 1969).

In November 2006, J. B. Fixler et al. measured the Newtonian constant of gravity, $G$, using a gravity gradiometer based on atom interferometry and reported a value of $G=6.693 \times 10^{-11} \mathrm{~m}^{3} \mathrm{~kg}^{-1} \mathrm{~s}^{-2}$, with a standard error of the mean of $\pm 0.027 \times 10^{-11}$ and a systematic error of $\pm 0.021 \times 10^{-11} \mathrm{~m}^{3} \mathrm{~kg}^{-1} \mathrm{~s}^{-2}$ (Fixler, 2007).

\section{Theoretical Discussion}

The gravitational constant $(G)$ is an empirical physical constant involved in the calculation of gravitational force between two bodies which appears in Sir Isaac Newton's law of universal gravitation given by in the vectorial notation (Newton, 1687).

$$
\vec{F}=-\frac{G m_{1} m_{2}}{r^{2}} \hat{r}
$$

where $m_{1}$ and $m_{2}$ are the mass of any two bodies, $r$ be the distance between them and $\hat{r}$ is the unit vector. The negative sign indicates that the nature of gravitational force is attractive. $\mathrm{G}$ denotes the gravitational constant and in Albert Einstein's theory of general relativity, given by (Einstein,1915).

$$
\begin{array}{r}
R_{i k}=1 / 2\left(g_{i k} R\right)=-\kappa T_{i k} \\
\text { Where } \kappa=8 \pi G / c^{4}
\end{array}
$$


The constancy for $G$ is experimentally confirmed in classical mechanics for the bodies where weak gravitational field is present, but theories that violate the strong equivalence principle by allowing for preferred locations may permit Newton's constant G varies (Stairs, 2003).

From equation (1), we have

$$
G=F r^{2} / m_{1} m_{2}
$$

Obviously, the gravitational constant is defined as the force of attraction acting between two bodies of unit mass placed at unit distance. From Newton's second law of motion, the force is closely related to the mass of the body as follow:

$$
\vec{F}=m \vec{a}
$$

where $\mathrm{m}$ is the mass of the body in classical mechanics. The variation of mass of a body due to change in velocity will affect the force. This affect should be associated with the gravitational constant $\mathrm{G}$ in the very strong gravitational field due to the compact bodies such as spinning black holes and neutron stars. According to the classical mechanics, the mass of body remains the same during either in motion or at the rest, but according to special theory of relativity, the mass of body varies with velocity as follows: (Bergmann,1969).

$$
m=\frac{m_{0}}{\sqrt{1-v^{2} / c^{2}}}
$$

Due to the variation of mass, the force acting on the body will also vary and the variation of force can be obtained by putting $\frac{m_{0}}{\sqrt{1-v^{2} / c^{2}}}$ instead of $m$ and hence, we have

$$
F^{\prime}=\frac{m_{0} a}{\sqrt{1-v^{2} / c^{2}}}
$$

Dividing eq ${ }^{\mathrm{n}}$ (7) by eq ${ }^{\mathrm{n}}$ (5) and solving, we have

$$
F^{\prime}=\frac{F}{\sqrt{1-v^{2} / c^{2}}}\left(\frac{m_{0}}{m}\right)
$$

For very low speed, $\mathrm{v}<<\mathrm{c}$ or $\mathrm{v} / \mathrm{c}<<1$, (Bergmann, 1969) then from $\mathrm{eq}^{\mathrm{n}}(6)$, we have

$$
m=m_{0}
$$

Putting the above value in equation (8), we have

$$
F^{\prime}=\frac{F}{\sqrt{1-v^{2} / c^{2}}}
$$

For two bodies of mass $m_{1}$ and $m_{2}$ placed at distance $r$ in the strong gravitational field \& weak gravitational field, the force of attraction is given respectively.

$$
\begin{gathered}
F^{\prime}=\frac{G^{\prime} m_{1} m_{2}}{r^{2}} \\
F=\frac{G m_{1} m_{2}}{r^{2}}
\end{gathered}
$$

Applying the $\mathrm{eq}^{\mathrm{n}}$ (11) and (12) into the $\mathrm{eq}^{\mathrm{n}}(10)$, we have

$$
G^{\prime} \frac{m_{1} m_{2}}{r^{2}}=G \frac{m_{1} m_{2}}{r^{2} \sqrt{1-v^{2} / c^{2}}}
$$

Solving the above equation, we obtain

$$
G^{\prime}=\frac{G}{\sqrt{1-v^{2} / c^{2}}}
$$

The equation (14) shows the variability of gravitational constant in the strong gravitational field like the gravitational field of black holes, neutron stars and quasars, depending upon the spinning velocity. E.S. Reich has shown graphically in his paper that the spinning rate of the super massive black holes begin from about $50 \%$ of the speed of light to $99 \%$ of the speed of light and some super massive black holes spin at more than $90 \%$ of the speed of light, which suggest that they gained their mass through major galactic mergers (Reich, 2013). It is also clear from the graph that no super massive black holes spin at rate below than $40 \%$ of the speed of light. On the basis of data regarding the speed of super massive black holes from $10 \%$ to $98 \%$ of the speed of light, we calculated the variability of gravitational constant with the help eq ${ }^{\mathrm{n}}(14)$ in the table 1.

\section{Data in support of variation of $G$}

There are so many constants of nature in which the gravitational constant $\mathrm{G}$ has a vital role in the study of gravitation. According to Newton's law of gravitation, this $\mathrm{G}$ is constant throughout the universe.

A relative distance between the Earth and Mars was accurately measured by taking thousands of range measurements between tracking stations of the Deep Space Network and Viking launders on Mars. From a least squares fit of the parameters of the solar system model to the data taken from various range measurements including those by Viking launders to Mars from July 1976 to July 1982, a bound on $\dot{G}$ is obtained: $\dot{G} / G=(2 \pm 4) \times 10^{-12} y r^{-1}$ (Hellings et al. 1983).

From the analysis of the data from 1969 to 1990, a bound on $\dot{G}$ is obtained: $\dot{G} / G=(0.1 \pm 10.4) \times 10^{-12} y r^{-1}$, while from the data from 1970 to 1994, 
$\dot{G} / G=(1 \pm 8) \times 10^{-12} y r^{-1}$. Recent analysis using the data up April 2004 yields $\dot{G} / G=(4 \pm 9) \times 10^{-13} y r^{-1}$. The uncertainty for $\dot{G} / G$ is improving rapidly since the sensitivity for the observations depends on the square of the time span (Chiba, 2011).

From the timing of the binary pulsar PSR 1913+16, a bound on $\dot{G}$ is obtained: $\dot{G} / G=(1.0 \pm 2.3) \times 10^{-11} y r^{-1}$ (Damour et al. 1988). When the effect of the variation in the gravitational binding energy induced by a change in $\mathrm{G}$ is taken into account, the above bound is somewhat weakened depending on the equation of state (Nordtvedt, 1990).

Jin Wang studied the astrophysical bounds on the change of the gravitational constant with time and found that $\dot{G} / G<10^{-12} y r^{-1}$ is the condition that has to be satisfied in order not to cause a conflict with the observations (Wang, 1991). In general, variation in $G$ are expected to occur on the timescale of the age of the universe, such that $\dot{G} / G \sim H_{0} \sim 0.7 \times 10^{-10} y r^{-1}$, where $\mathrm{H}_{0}$ is the Hubble constant and the strong equivalence principle violating time-variable $\mathrm{G}$ would be expected to alter the properties of neutron stars and white dwarfs and to affect binary orbits(Stairs, 2003).

\section{Table}

\begin{tabular}{|c|c|c|c|c|}
\hline \multirow[b]{2}{*}{ Sl. No } & \multicolumn{4}{|c|}{ Variability of Newton's constant in the strong gravitational field. } \\
\hline & $\begin{array}{l}\% \text { speed of super } \\
\text { massive black } \\
\text { holes, Neutron } \\
\text { stars, Quasars of } \\
\text { the speed of light }\end{array}$ & $\begin{array}{l}\text { Speed of Super massive black } \\
\text { holes, Neutron stars, Quasars } \\
(\mathrm{m} / \mathrm{s})\end{array}$ & $\begin{array}{l}\text { Newton's constant } G \\
\left(\mathrm{~m}^{3} \mathrm{Kg}^{-1} \mathrm{~s}^{-2}\right)\end{array}$ & $G^{\prime}={\frac{G}{\sqrt{1-v^{2} / c^{2}}}}^{\left(\mathrm{m}^{3} \mathrm{Kg}^{-1} \mathrm{~s}^{-2}\right) .}$ \\
\hline 1 & 3 & $0.9 \times \times 10^{7}$ & $6.67384 \times 10^{-11}$ & $6.6708 \times 10^{-11}$ \\
\hline 2 & 4 & $1.2 \times \times 10^{7}$ & $6.67384 \times 10^{-11}$ & $6.6782 \times 10^{-11}$ \\
\hline 3 & 5 & $1.5 \times 10^{7}$ & $6.67384 \times 10^{-11}$ & $6.682 \times 10^{-11}$ \\
\hline 4 & 10 & $3.0 \times 10^{7}$ & $6.67384 \times 10^{-11}$ & $6.696 \times 10^{-11}$ \\
\hline 5 & 15 & $4.5 \times 10^{7}$ & $6.67384 \times 10^{-11}$ & $6.750 \times 10^{-11}$ \\
\hline 6 & 20 & $6.0 \times 10^{7}$ & $6.67384 \times 10^{-11}$ & $6.810 \times 10^{-11}$ \\
\hline 7 & 25 & $7.5 \times 10^{7}$ & $6.67384 \times 10^{-11}$ & $6.894 \times 10^{-11}$ \\
\hline 8 & 30 & $9.0 \times 10^{7}$ & $6.67384 \times 10^{-11}$ & $7.007 \times 10^{-11}$ \\
\hline 9 & 35 & $1.05 \times 10^{8}$ & $6.67384 \times 10^{-11}$ & $7.125 \times 10^{-11}$ \\
\hline 10 & 40 & $1.20 \times 10^{8}$ & $6.67384 \times 10^{-11}$ & $7.280 \times 10^{-11}$ \\
\hline 11 & 45 & $1.35 \times 10^{8}$ & $6.67384 \times 10^{-11}$ & $7.473 \times 10^{-11}$ \\
\hline 12 & 50 & $1.50 \times 10^{8}$ & $6.67384 \times 10^{-11}$ & $7.706 \times 10^{-11}$ \\
\hline 13 & 55 & $1.65 \times 10^{8}$ & $6.67384 \times 10^{-11}$ & $7.992 \times 10^{-11}$ \\
\hline 14 & 60 & $1.80 \times 10^{8}$ & $6.67384 \times 10^{-11}$ & $8.342 \times 10^{-11}$ \\
\hline 15 & 65 & $1.95 \times 10^{8}$ & $6.67384 \times 10^{-11}$ & $8.785 \times 10^{-11}$ \\
\hline 16 & 70 & $2.10 \times 10^{8}$ & $6.67384 \times 10^{-11}$ & $9.345 \times 10^{-11}$ \\
\hline 17 & 75 & $2.25 \times 10^{8}$ & $6.67384 \times 10^{-11}$ & $10.091 \times 10^{-11}$ \\
\hline 18 & 80 & $2.40 \times 10^{8}$ & $6.67384 \times 10^{-11}$ & $11.120 \times 10^{-11}$ \\
\hline 19 & 85 & $2.55 \times 10^{8}$ & $6.67384 \times 10^{-11}$ & $12.671 \times 10^{-11}$ \\
\hline 20 & 90 & $2.70 \times 10^{8}$ & $6.67384 \times 10^{-11}$ & $15.356 \times 10^{-11}$ \\
\hline 21 & 95 & $2.85 \times 10^{8}$ & $6.67384 \times 10^{-11}$ & $22.816 \times 10^{-11}$ \\
\hline 22 & 98 & $2.94 \times 10^{8}$ & $6.67384 \times 10^{-11}$ & $47.109 \times 10^{-11}$ \\
\hline
\end{tabular}




\section{Graph}

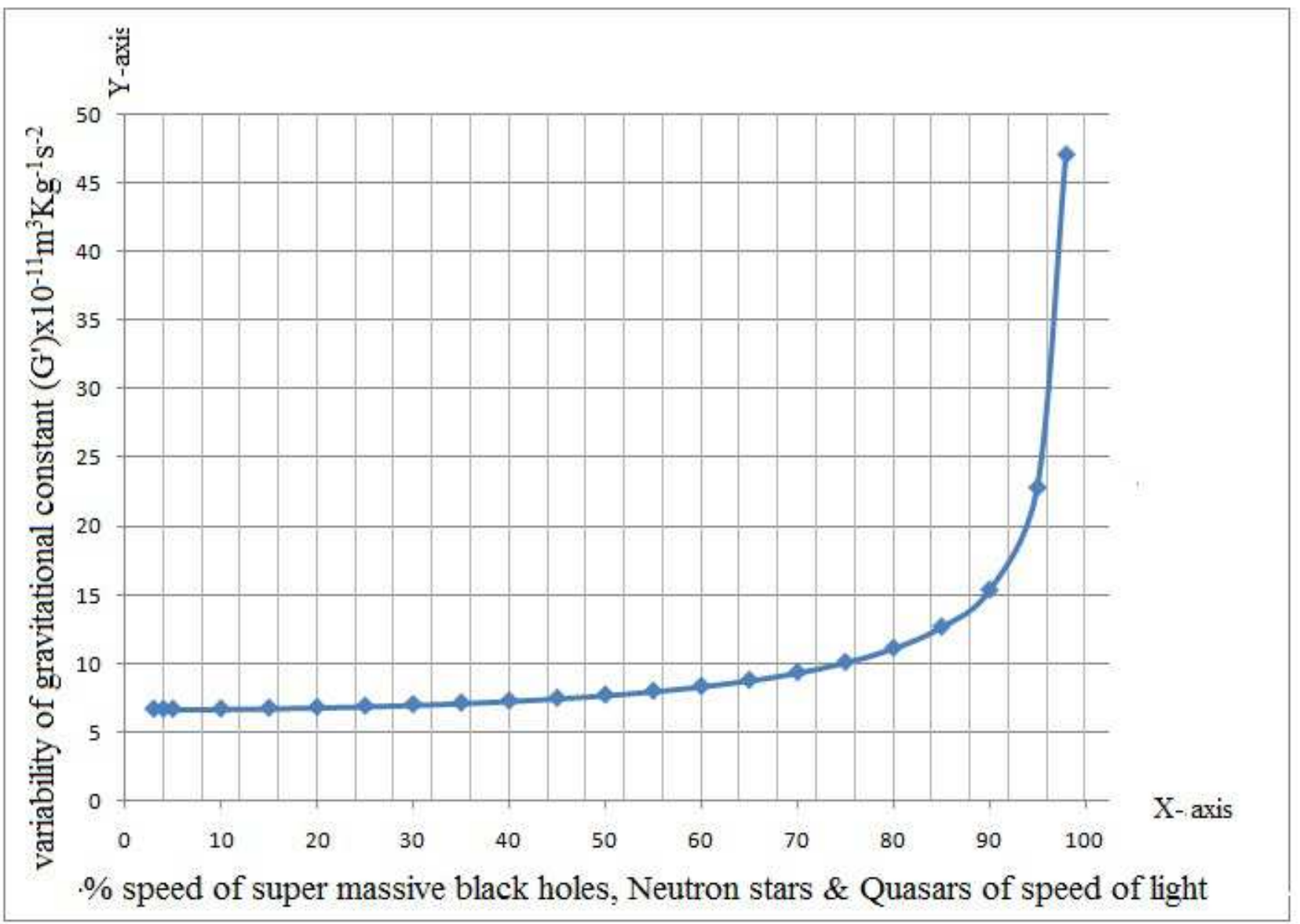

Figure 1: The graph plotted between variability of gravitational field $G^{\prime}$ and \% speed of super massive black holes, neutron stars and quasars of speed of light.

\section{Results and Discussion}

In the present work, we have derived the formula for the variation of the gravitational constant given by $G^{\prime}=G / \sqrt{1-v^{2} / c^{2}}$ in very strong gravitational field of the compact bodies like black holes and neutron stars applying special relativity and Newton's law of gravitation for two bodies where $\mathrm{v}$ be the velocity of spinning compact bodies like black holes, neutron stars etc. and $\mathrm{c}$ be the velocity of light. In the present work, we have also calculated the variability of the gravitational constant $(G)$ at different speed of the spinning of the black holes, neutron stars and quasars. It has been observed from the table and graph that the variability of the gravitational constant $(G)$ increases gradually with the increase of the speed of the black holes, neutron stars and quasars up to $80 \%$ of the speed of light and after $85 \%$, the variability of the gravitational constant $(\mathrm{G})$ increases rapidly up to $100 \%$. This shows that for the super massive black holes, neutron stars and quasars spinning almost equal to the speed of light gain maximum gravity.

This means that the speed is also factor governing the force of gravity addition to the mass, because new theory of gravitation is dependent on the speed of bodies which states that the factor joining the whole Solar system and galaxies all in one piece would have to have higher speed than the velocity of light. At present, no higher speed is known than the velocity of light, thus the phenomenon ascribed to gravitation must have other form than that know currently as the phenomenon of gravitational pull (Borowski, 2012).

\section{Conclusion}

In course of the present research work, we can draw the following conclusions such as:

(i) The super massive black holes, neutron stars and quasars spinning with almost equal to the speed of light gain maximum gravity than that of the gravity of super massive black holes, neutron stars and quasars spinning with almost up to $80 \%$ of the speed of light.

(ii) The speed is also factor governing the force of gravity in addition to the mass

(iii) The formula $G^{\prime}=G / \sqrt{1-v^{2} / c^{2}}$ holds good for the gravity of super massive black holes, neutron stars and quasars spinning with the speed comparable to the speed of light. 


\section{Acknowledgement}

The authors are grateful to the referee for pointing out the errors in the original manuscript and making constructive suggestions. The authors are also grateful to Dr. (Prof.) Gopi Kant Jha, the former H. O. D, University Department of Physics, L. N. M. U. Darbhanga (India), Dr. Neeraj Pant, Associate Professor, Department of Mathematics, N. D. A. Khadakwasala, Pune and Prof. M.S.H. John, Principal \& V. K. Mishra, H.O.D. Physics, Marwari College Bhagalpur for their inspiration and motivation.

\section{References}

[1] I. Newton,: "The Principia (The mathematical principles of Natural knowledge)”, 1667.

[2] A. Einstein, Preuss, Akad. Wiss, Berlin, Sitzber, pp,778, 831, and 844 (1915).

[3] Brush, Stephen G.; Holton, Gerald James (2001), Physics, the human adventure: from Copernicus to Einstein and beyond, New Brunswick, N.J: Rutgers University Press, p. 137, ISBN 0-8135-2908-5

[4] P.G. Bergmann,: "Introduction to the Theory of Relativity". Prentice Hall of India, New Delhi (1969).
[5] .J. B. Fixler; G. T. Foster; J. M. McGuirk; M. A. Kasevich (2007-01-05), "Atom Interferometer Measurement of the Newtonian Constant of Gravity" Science 315 (5808): 74-77, Doi:10.1126/science.1135459 PMID 17204644, 2007.

[6] Jin Wang: "Astrophysical constraints on the gravitational constant", Astrophysics and Space Science, 184, 31-36(1991).

[7] Ingrid H. Stairs: "Testing General Relativity with Pulsar Timing.” Living Reviews in Relativity, 2003.

[8] Tomasz Borowski: "The new theory of gravitation representing the movement of planets." International Letters of Chemistry, Physics and Astronomy 1 (2012) 1-5.

[9] Eugenie Samuel Reich: "Spin rate of black holes pinned down.” Nature, Vol.500,p-135, Macmillan Publishing limited, Aug 2013.

[10] Takeshi Chiba: "The Constancy of the Constants of Nature: Updates." Progress of Theoretical Physics, Vol.126, No. 6, Dec. 2011.

[11] K. Nordtvedt: Physical Review Letter, 65, (1990),953.

[12] T. Damour, G.W. Gibbons and J. H. Taylor, Physical Review Letter,61, (1988), 1151. 